\title{
Adjunctive favipiravir for severe COVID-19: a retrospective observational study of the first 41 patients in Thailand
}

\author{
Wisit Prasithsirikul', Krit Pongpirul2,3,*, Phuwanat Sakornsakolpat', Chuti Burana', \\ Phanupong Phutrakool ${ }^{2}$, Wannarat A. Pongpirul'
}

Abstract

Background: Favipiravir is a promising drug for COVID-19, but evidence from a robust clinical trial is limited.

Objective: To describe the demographics, clinical characteristics, and various antiviral treatment regimens (with and without favipiravir) of patients with severe and nonsevere COVID-19.

Method: We conducted a retrospective observational study in all COVID-19 patients admitted at Bamrasnaradura Infectious Diseases Institute (BIDI) from January 8 to March 30, 2020. We compared the demographics, clinical characteristics, and various antiviral treatment regimens of 12 severe and 29 nonsevere COVID-19 patients in Thailand. Results: Adjunctive favipiravir was given to only severe cases. The median length of hospitalization of patients either receiving favipiravir or not receiving favipiravir was not significantly different $(P=0.8549)$, but those who received adjunctive favipiravir became reverse transcriptase-polymerase chain reaction negative 2 days sooner than the other group (median: 6 days vs. 8 days; $P=0.1125$ ).

Conclusion: The findings suggested that adjunctive favipiravir might not be effective for patients with severe COVID-19, but further studies with larger sample sizes are needed.

Keywords: COVID-19; favipiravir; SARS-Cov-2

Favipiravir, an RNA-dependent RNA polymerase inhibitor, is one of the promising drugs investigated for the treatment of coronavirus disease 2019 (COVID-19) [1-3]. It was approved for the treatment of COVID-19 in China, and preliminary results from an ongoing open-label, nonrandomized controlled trial of nonsevere patients suggested that oral favipiravir 1,600 mg twice daily on Day 1, followed by $600 \mathrm{mg}$ twice daily showed a superior antiviral action than that of oral lopinavir $400 \mathrm{mg}$ and ritonavir $100 \mathrm{mg}$ twice daily [4]. A randomized controlled trial recently published in a preprint found that compared to arbidol, favipiravir did not significantly improve clinical recovery at 1 week [5].

Given the present pandemic, evidence on the clinical effectiveness of each antiviral regimen in real-life settings,

*Correspondence to: Krit Pongpirul, Department of Preventive and Social Medicine, Faculty of Medicine, Chulalongkorn University, Bangkok 10330, Thailand, e-mail: krit.po@chula.ac.th

'Bamrasnaradura Infectious Diseases Institute, Department of Disease Control, Ministry of Public Health, Nonthaburi 11000, Thailand

2Department of Preventive and Social Medicine, Faculty of Medicine, Chulalongkorn University, Bangkok 10330, Thailand

${ }^{3}$ Department of International Health, Johns Hopkins Bloomberg School of Public Health, MD 21205, USA

Ә Open Access. ๑ 2020 Prasithsirikul et al., published by Sciendo. (c) Br-Nc-ND This work is licensed under the Creative Commons Attribution NonCommercial-NoDerivatives 4.0 License. 
especially from less-developed countries, is essential yet limited. Various combinations of antivirals have been used simultaneously along with other supportive therapies, resulting in a more difficult context for evidence-based clinical decision-making. As potential drugs have joined the personal protective equipment to become scarce resources for fighting COVID-19, price and availability concerns are inevitable.

Thailand is one of the countries that have been affected by the novel coronavirus (severe acute respiratory syndrome coronavirus 2 or SARS-CoV-2) [6, 7] but was fortunate enough to procure favipiravir, which is available only in Asia. Given its price and availability, the Thai Food and Drug Administration authorized the use of favipiravir as an adjunctive antiviral therapy to only severe COVID-19 patients under the clinical care system of the Department of Disease Control, Ministry of Public Health.

This report aims to describe the demographics, clinical characteristics, and various antiviral treatment regimens (with vs. without favipiravir) of patients with severe and nonsevere COVID-19 treated at the Bamrasnaradura Infectious Diseases Institute (BIDI), Thailand.

\section{Methods}

This study was approved by the Institutional Review Board of Bamrasnaradura Infectious Diseases Institute, Department of Disease Control, Ministry of Public Health (certificate of approval no. S012h_63_ExPD). We used the "Strengthening the Reporting of Observational Studies in Epidemiology (STROBE)" cohort checklist when writing our report [8].

In this retrospective observational study, we reviewed all 41 COVID-19 patients confirmed by a positive result on a reverse transcriptase-polymerase chain reaction (RT-PCR) assay of a nasopharyngeal swab specimen. They were admitted at Bamrasnaradura Infectious Diseases Institute (BIDI), Nonthaburi, Thailand, from January 8 to March 30, 2020. One major source of bias is that favipiravir was allowed only for severe COVID-19 cases. Various medication regimens were used based on the dosages summarized in Table 1. Disease severity was classified into three levels: 1 (Mild), 2 (Moderate; abnormal chest $\mathrm{X}$-ray and respiratory rate $\leq 24$ breaths per minute, and 3 (Severe; progressive chest X-ray or respiratory failure). Treatment efficacy was assessed by the time of viral clearance and length of hospital stay.

Descriptive statistics were used to summarize the data; results are reported as means and standard deviations, or medians and interquartile ranges, as appropriate. Categorical variables are presented as counts and percentages. Wilcoxon rank sum test was used to compare the length of stay and days from treatment initiation to negative RT-PCR result. The analysis was performed with Stata/MP 15.1 software (StataCorp LP, College Station, TX, USA).

\section{Results}

Forty-one COVID-19 patients were included in this study. Their mean age was 45 years, and $40 \%$ were female. Twenty-four patients $(58.5 \%)$ had no pneumonia (disease severity level 1 ) whereas three patients $(7.3 \%)$ were mechanically ventilated and dead (Table 2). Thirteen patients $(31.7 \%)$ received at least $\mathrm{LPV} / \mathrm{r}$ or DRV/r, whereas 12 patients (29.3\%) also received CQ/HCQ. Ten patients $(22.0 \%)$ received adjunctive favipiravir, one of whom also received remdesivir.

The median lengths of hospitalization of patients either receiving favipiravir or not receiving favipiravir were not

Table 1. COVID-19 medication dosages at the Bamrasnaradura Infectious Diseases Institute (BIDI), Thailand

\begin{tabular}{llll}
\hline Medication & Day $\mathbf{1}$ & Days 2-5 & Days 6-10 \\
\hline Favipiravir (200 mg) PO & 8 tablets twice a day & 3 tablets twice a day & 3 tablets twice a day \\
Lopinavir/ritonavir (LPV/r) $(200 \mathrm{mg} / 50 \mathrm{mg})$ PO & 2 tablets twice a day & 2 tablets twice a day & 2 tablets twice a day \\
Darunavir/ritonavir (DRV/r) $(600 \mathrm{mg} / 100 \mathrm{mg})$ PO & 1 tablet twice a day & 1 tablet twice a day & 1 tablet twice a day \\
Darunavir (DRV) $(600 \mathrm{mg})$ PO & $\begin{array}{l}1.5 \text { tablets daily or } 1 \text { tablet } \\
\text { twice a day }\end{array}$ & $\begin{array}{l}1.5 \text { tablets daily or } 1 \text { tablet } \\
\text { twice a day }\end{array}$ & $\begin{array}{l}1.5 \text { tablets daily or } 1 \text { tablet } \\
\text { twice a day }\end{array}$ \\
Ritonavir (r) (100 mg) PO & 1 tablet daily or 1 tablet twice & 1 tablet daily or 1 tablet twice & 1 tablet daily or 1 tablet twice \\
Chloroquine (CQ) (250 mg) PO & a day & a day & a day \\
Hydroxychloroquine (HCQ) (200 mg) PO & $1-2$ tablets twice a day & $1-2$ tablets twice a day & $1-2$ tablets twice a day \\
Remdesivir IV & 1 tablet twice a day & 1 tablet twice a day & 1 tablet twice a day
\end{tabular}

IV, intravenously; PO, orally. 
significantly different $(P=0.86)$, but those who received adjunctive favipiravir became RT-PCR negative 2 days sooner than the other group (median: 6 days vs. 8 days; $P=0.11$ ) (Table 3). No major adverse drug reactions were observed.

Table 2. Characteristics of 41 patients with COVID-19 in the study

\begin{tabular}{lc}
\hline Characteristics & Mean/frequency \\
\hline Age, years, $\mathrm{n}(\mathrm{SD})$ & $45(15.5)$ \\
Females, $\mathrm{n}(\%)$ & $17(41.5)$ \\
Health workers, $\mathrm{n}(\%)$ & $1(2.4)$ \\
Comorbidities, $\mathrm{n}(\%)$ & \\
Hypertension & $6(14.6)$ \\
Dyslipidemia & $2(4.9)$ \\
Diabetes mellitus & $2(4.9)$ \\
Cardiac diseases & $3(7.3)$ \\
Chronic liver diseases & $3(7.3)$ \\
Chronic hematologic diseases & $1(2.4)$ \\
Rheumatologic diseases & $1(2.4)$ \\
Disease severity level, $\mathrm{n}(\%)$ & \\
Mild & $24(58.5)$ \\
Moderate & $5(12.2)$ \\
Severe & $12(29.3)$ \\
On ventilator, $\mathrm{n}$ (\%) & $3(7.3)$ \\
Deaths, $\mathrm{n}(\%)$ & $3(7.3)$ \\
Medication profile, $\mathrm{n}$ (\%) & $13(31.7)$ \\
LPV/r or DRV/r & $12(29.3)$ \\
LPV/r or DRV/r + CQ or HCQ & $9(22.0)$ \\
LPV/r or DRV/r + CQ or HCQ + favipiravir & $1(2.4)$ \\
LPV/r or DRV/r + CQ or HCQ + favipiravir & \\
+ remdesivir & $6(14.6)$ \\
Other or no specific medications & \\
\hline & \\
\hline
\end{tabular}

$\mathrm{LPV} / \mathrm{r}$, lopinavir/ritonavir; DRV/r, darunavir/ritonavir; $\mathrm{CQ}$, chloroquine; $\mathrm{HCQ}$, hydroxychloroquine.

\section{Discussion}

Findings from our experience suggest that adjunctive favipiravir might not be effective for patients with severe COVID19. Given the lack of evidence on the clinical effectiveness of favipiravir on COVID-19 at the initial phase of our study, along with the limited availability of the medications as mentioned earlier, favipiravir was reserved for only severe patients in Thailand. We believe that the clinical outcomes could have been better had favipiravir been administered earlier, as suggested by the potential superior clinical efficacy of adding favipiravir to $\mathrm{LPV} / \mathrm{r}$ (4).

Initially, we tried to administer favipiravir for only 5 days, which was extended to 10 days based mainly on clinical judgment and partly on the slightly improved chest X-ray findings. This is a good example of the real-life practices that were based mainly on clinical judgment because no standardized treatment protocols were available.

The major limitations of our observational study are as follows: (i) favipiravir was allowed only for severe COVID-19 cases, which is a potential source of bias; and (ii) the relatively small size of the sample, which limited generalization of the findings. This could have been improved by adopting a more robust design, such as a randomized controlled trial with larger sample size. Furthermore, we were not able to collect a comprehensive set of clinical outcomes, such as computed tomography of the chest, quantitative viral assessment, or other blood parameters. However, an early experience of a less-developed country might be beneficial for many other similar contexts.

\section{Conclusion}

Adjunctive favipiravir might not be effective for severe COVID-19 patients, but further studies with larger sample sizes are needed.

Table 3. Outcomes in COVID-19 patients treated with and without favipiravir

\begin{tabular}{lccc}
\hline Outcomes & Overall $(\mathbf{n}=\mathbf{4 1})$ & With favipiravir $(\mathbf{n}=\mathbf{1 0})$ & Without favipiravir $(\mathbf{n}=\mathbf{3 1})$ \\
\hline Disease severity level, $\mathrm{n}(\%)$ & & & $\boldsymbol{P}$ \\
Mild & $24(58.5 \%)$ & $0(0.0 \%)$ & $24(77.4 \%)$ \\
Moderate & $5(12.2 \%)$ & $0(0.0 \%)$ & $5(16.1 \%)$ \\
Severe & $12(29.3 \%)$ & $10(100.0 \%)$ & $2(6.5 \%)$ \\
Length of stay, median (IQR) & $9.0(7.0,14.0)$ & $8.0(7.3,13.0)$ & $10.0(7.0,12.5)$ \\
Days from start of treatment to PCR-negative & $7.0(5.0,10.0)$ & $6.0(4.3,8.5)$ & $8.00(6.0,10.0)$ \\
result, median (IQR) & & & 0.86 \\
\hline
\end{tabular}

RT-PCR, reverse transcriptase-polymerase chain reaction; SARS-CoV-2, severe acute respiratory syndrome coronavirus 2; IQR, interquartile ranges. 
Author contributions. WP and KP contributed substantially to the conception and design of this study. WP, PS, $\mathrm{CB}$, and WAP contributed substantially to the acquisition of data. KP, PS, and PP analyzed and interpreted the data. WP, KP, PS, PP drafted the manuscript. CB and WAP contributed substantially to its critical revision. All the authors approved the final version submitted for publication and take responsibility for the statements made in the published article.

Conflict of interest statement. The authors have each completed and submitted an International Committee of Medical Journal Editors Uniform Disclosure Form for Potential Conflicts of Interest. None of the authors discloses any potential or actual conflict of interest. No financial or nonfinancial benefits have been or will be received from any party related directly or indirectly to the subject of this article.

Data sharing statement. The data sets generated or analyzed during the current study are available from the corresponding author on reasonable request.

\section{References}

[1] Delang L, Abdelnabi R, Neyts J. Favipiravir as a potential countermeasure against neglected and emerging RNA viruses. Antiviral Res. 2018; 153:85-94.

[2] Dong L, Hu S, Gao J. Discovering drugs to treat coronavirus disease 2019 (COVID-19). Drug Discov Ther. 2020; 14:58-60.

[3] Du YX, Chen XP. Favipiravir: pharmacokinetics and concerns about clinical trials for 2019-nCoV infection. Clin Pharmacol Ther. 2020; 108:242-7. doi: 10.1002/cpt.1844.

[4] Cai Q, Yang M, Liu D, Chen J, Shu D, Xia J, et al. Experimental treatment with Favipiravir for COVID-19: an open-label control study. Engineering (in print). 2020. doi: 10.1016/j.eng.2020.03.007. Online ahead of print.

[5] Chen C, Zhang Y, Huang J, Yin P, Cheng Z, Wu J, et al. Favipiravir versus arbidol for COVID-19: a randomized clinical trial. medRxiv 2020.03.17.20037432. doi: 10.1101/2020.03.17.20037432

[6] Pongpirul W, Mott J, Woodring J, Uyeki T, MacArthur J, Vachiraphan A, et al. Clinical characteristics of initial hospitalized COVID-19 patients in Thailand. Emerg Infect Dis. 2020; 26:1580-5.

[7] Pongpirul WA, Pongpirul K, Ratnarathon AC, Prasithsirikul W. Journey of a Thai taxi driver and novel coronavirus. N Engl J Med. 2020; 382:1067-8.

[8] von Elm E, Altman DG, Egger M, Pocock SJ, Gotzsche PC, Vandenbroucke JP, et al. The strengthening the reporting of observational studies in epidemiology (STROBE) statement: guidelines for reporting observational studies. PLoS Med. 2007; 4:e296. 